\title{
Strates
}

STRATES Matériaux pour la recherche en sciences sociales

$4 \mid 1989$

Dossier : images réfléchies. Paroles d'un paysan révolutionnaire

\section{Publicité et géographie : paysages, images et discours}

Didier Mendibil

\section{(2) OpenEdition \\ Journals}

Édition électronique

URL : http://journals.openedition.org/strates/4162

DOI : $10.4000 /$ strates.4162

ISSN : $1777-5442$

Éditeur

Laboratoire Ladyss

Édition imprimée

Date de publication : 31 décembre 1989

ISSN : 0768-8067

Référence électronique

Didier Mendibil, «Publicité et géographie : paysages, images et discours », Strates [En ligne], 4| 1989,

mis en ligne le 19 mai 2008, consulté le 08 septembre 2020. URL : http://journals.openedition.org/

strates/4162 ; DOI : https://doi.org/10.4000/strates.4162

Ce document a été généré automatiquement le 8 septembre 2020.

Tous droits réservés 


\title{
Publicité et géographie : paysages, images et discours
}

\author{
Didier Mendibil
}

1 La problématique du paysage a été réactualisée lors de la récente « crise » d'identité de la géographie, à la fois pour légitimer un champ opératoire particulier et affirmer une des spécificités de la discipline.

2 Dans le même temps - à la charnière des années 1970-80 - la publicité s'est intéressée aux paysages : il s'agissait, avec ce nouveau support de communication, de s'adapter à une sensibilité écologiste croissante - en utilisant les images valorisées de la ruralité par exemple -, et de soutenir des secteurs économiques sensibles au surenchérissement du pétrole : l'automobile, le tourisme lointain, etc.

3 Et, depuis, la place des paysages n'a cessé de grandir dans la sensibilité de l'opinion publique comme dans les préoccupations des «décideurs». A tel point qu'ils constituent aujourd'hui l'un des enjeux quotidiens de l'aménagement du territoire et, souvent, le «lieu », voire l'instrument de cristallisation de certains conflits sociaux et politiques. Il est donc important de se demander pourquoi et surtout comment on parle des paysages aujourd'hui.

4 Dans ce domaine, publicité et géographie tiennent des discours que tout semble opposer puisque l'une conduit une stratégie marchande alors que l'autre poursuit un projet scientifique. Cependant, confronter ces deux types de discours c'est, d'une certaine manière, entrer dans le débat public qui les confronte dans la société. C'est faire aussi qu'ils s'éclairent mutuellement, notamment en ce qui concerne l'établissement de liens entre le domaine de la perception et celui du langage, ou, plus simplement, entre un texte et une image.

Ouvrir ici ce dialogue, c'est montrer que, face aux paysages, publicistes et géographes ${ }^{1}$ déploient une pratique qui n'est « ni tout à fait la même, ni tout à fait une autre ». C'est aussi ouvrir la vaste question du rôle des images - visibles ou mentales - dans l'organisation de l'espace géographique.

Des discours sur le paysage 
6 Deux exemples concrets vont permettre de poser le problème des discours portés sur des paysages: une échelle comparable - pas trop grande-, un point de vue panoramique, une perspective jalonnée de repères identifiables, des lignes visibles complexes où dominent les courbes, un contact entre espace habité et espace «naturel » souligné par des contrastes lumineux bien marqués, autorisent à qualifier ces photographies de "paysages » au sens géographique du terme et incitent à une comparaison de ces deux images publicitaires.

Un paysage riche de significations : le village grec

7 Le premier document se prête bien à une stratégie publicitaire car il déploie instantanément tous les charmes que la polysémie peut offrir au regard d'une subjectivité. Si l'on reprend le cadre analytique proposé par Michel COLLOT ${ }^{2}$, la perspective sémiotique y découvre un "beau (paysage) dont les structures propres (dues au relief, à l'éclairage) renforcent l'organisation (sélective et relationnelle) que l'intelligence perceptive impose à tout objet spatial». Sont livrés au regard les couples terre/mer, montagne/vallée, lieux habités/lieux "sauvages », ombre/lumière... etc., articulés en une métonymie (exhaustive!) du monde: un microcosme. Dans la perspective psychanalytique l'on découvrira une profusion de formes où peuvent se nicher les fantasmes, individuels et collectifs, les connotations et les symboles (forteresse dressée, village niché, imbrication de l'eau et des rochers, etc.). Quant à la perspective phénoménologique, elle retiendra l'efficacité d'un punctum, cette petite maison isolée - ouverte sur le monde, face au village que l'on voit sans être vu - par laquelle ce paysage devient un espace du projet individuel, une mise en scène du cadre existentiel de l'homme : la coexistence moi/autrui, individu/humanité, unité/tout, présent/passé. A contempler ce paysage, une cascade de mots vient à l'esprit sur un mode quasi poétique. Il a tout pour plaire! (cf. Doc. en annexe. La mer a un pays : la Grèce.)

8 Le discours des géographes traditionnels - pour simplifier, disons ceux du XIX ${ }^{e}$ siècle $-y$ aurait trouvé matière à quelques pages lyriques sur les atouts et contraintes d'un tel site, élevé au rang d'archétype de la cité grecque, sobre et jalouse de son indépendance. Il aurait développé l'idée de la nécessaire ouverture sur l'espace maritime. Un discours contemporain noterait plutôt le potentiel touristique du site et soulignerait les problèmes posés par le passage d'une économie maritime à une économie touristique. Ce qui l'intéresserait surtout, ce serait le problème de l'inversion des flux : de terre/ mer à mer/terre. Dans tous les cas, ce paysage ne serait considéré qu'en tant qu'élément d'information à intégrer dans un ensemble de documents plus vaste seul susceptible de permettre une étude sérieuse.

Qu'en fait la publicité ? En apposant, sous la photographie, l'étrange formule " la mer a un pays : la Grèce ", elle laisse libre cours à l'effet polysémique du paysage qui fonctionne de façon autonome, c'est-à-dire sans que le texte ne l'oriente. Mais, dans la formule, la transgression de la syntaxe habituelle, attendue, cristallise sur le mot Grèce le sentiment d'idéalité qui se dégage de ce paysage "rêvé ». Elle en fait ainsi une image symbolique qui envahit le signifiant "Grèce " et renforce la mythologie touristique associée à ce pays. La signification change d'échelle : par un procédé métonymique et une inversion syntaxique, ce village grec devient la cité grecque moderne, une image mythique. C'est la « rencontre » entre le texte et l'image qui produit cet effet de sens. Le discours géographique, lui-même, aurait-il résisté à une telle utilisation de ce paysage pour illustrer son propos ? N’en aurait-il pas fait, volontiers, un « idéal-type », 
au sens de Weber? Ou un " cliché ", une carte postale, si le public visé n'est pas spécialiste? On peut se poser la question.

Un paysage mis à plat : la résidence « Panoramis »

10 Le deuxième document illustre un traitement publicitaire bien différent. Le paysage n'a aucune autonomie. Sa polysémie est réduite à une complète instrumentalité : tout signe visible est étiqueté d'une fonction, tout est réduit au même dénominateur technique. Ce paysage est quantifiable: en argent comptant! C'est un codage, une carte développée dans la troisième dimension... celle du raisonnement ! Seule l'architecture sinueuse peut/doit, ici, constituer l'objet d'un désir, assez conventionnel « au demeurant ». Les mots tarissent vite sur ce paysage : il n'est pas fait pour cela.

11 Le traitement textuel contrôle la polysémie de l'image en imposant une lecture fonctionnaliste. Le texte juxtapose, terme à terme, des éléments qualitatifs - «une superbe architecture ", "le dépaysement » (!), "des appartements luxueux », "à qualité égale » - à des éléments quantifiables ou techniques "...dans un site unique», "...dans la proximité ", "...entièrement équipés ", "tellement moins chers... ». Le texte impose l'axe de lecture subjectivité/objectivité au profit du second terme. La réalité de ce paysage est seconde : c'est une fiction créée de toutes pièces par le discours qu'il est sensé illustrer «preuves à l'appui ».

12 Qu'en aurait fait une approche géographique? Celle qui dominait la question des paysages dans les années 1950-60 aurait pu procéder un peu de la même manière. Certains manuels de géographie le feraient encore même s'ils y ajoutent quelques considérations sur l'urbanisation de la banlieue. On peut voir dans le traitement imposé à ce paysage la caricature - exagérée, je l'accorde - de l'approche fonctionnaliste des paysages propre à une certaine géographie. Elle serait assez proche, d'ailleurs, de la manière dont certains paysagistes abordent les problèmes d'aménagement urbain.

Des « modèles réduits »

13 De l'analyse de ces deux exemples de paysages constitués en objets de désir, retenons deux traitements publicitaires situés aux limites de l'axe subjectivité/objectivité. Dans un cas, le « réel » est livré dans toutes ses dimensions signifiantes; dans l'autre, il est réduit à un "message " monosémique qui le réifie. Le paysage réel subit donc une double réduction: la première est opérée par le cadrage qui crée l'image (et par d'autres opérations d'ordre technique : couleur, tirage,...). Les géographes ont appris à se méfier de cela ; ils raisonnent à plusieurs échelles avant d'avancer des conclusions. La seconde réduction est due à l'introduction d'un texte, d'un commentaire, qui oriente la lecture de l'image. Dans certains cas, le texte et l'image se renforcent mutuellement, et le «message » que crée cette interaction est jugé « riche », c'est-à-dire susceptible d'exercer un puissant effet de sens, de conviction sur qui le reçoit. Ce mécanisme de création d'images à la fois visuelles et mentales est à la base d'un processus que résume le concept d'« Imaginaction" auquel nous allons recourir tout à l'heure. Dans d'autres cas, c'est l'un des signifiants qui tire le sens à lui : l'imaginaction est alors négative ou occultante. Deux stratégies du sens: l'exacerbation de la polysémie qui dilue la signification mais élargit son impact, ou la réduction de la polysémie qui force le sens. (cf. Doc. en annexe. Une superbe architecture dans un site unique.)

14 Ces procédures de réduction du sens sont bien repérables dans le cas des messages publicitaires: c'est leur raison d'être, et ils ne s'en cachent pas. Mais le discours scientifique procède aussi à des réductions du sens. Souvent, il le fait par souci d'objectivité, mais est-ce toujours légitime ou conscient? Une analyse des pratiques 
publicitaires devrait donc préparer utilement celle de la pratique géographique des paysages.

Modalités de la publicité paysagère

Sur un ensemble de « réclames » publiées dans des hebdomadaires entre 1978 et 1981 ont été étudiés ${ }^{3}$ :

- le type de relation établie entre le paysage choisi et le produit concerné,

17 - la forme de signification propre au paysage,

18 - le type de relation existant entre le texte et l'image photographique.

19 Cette analyse a permis de distinguer un nombre limité d'approches "paysagères » que nous résumerons en quatre modes principaux :

Le mode de la dénotation

De nombreuses publicités exposent un paysage comme objet de désir puisqu'il s'agit, au sens propre, du produit à consommer. Le tourisme offre à profusion ces paysages souvent stéréotypés (mer, sable, soleil, palmiers, maisons rurales) qui, en fait, signifient "vacances ». Le texte se limite souvent à une dénomination (La Corse, la Manche,...) ou même à une exclamation. Ou alors, c'est le dessin d'un paysage qui est préféré à sa reproduction photographique, sans doute pour que la correspondance entre le texte et l'image se fasse terme à terme, dans un souci évident de démonstration et d'exhaustivité. D'où un effet redondant et systématique qui fait penser aux documents pédagogiques illustrant certains manuels de géographie destinés aux jeunes élèves. Si le procédé pédagogique est efficace, il donne des messages pauvres. Comme l'écriture, le dessin réduit la polysémie du paysage pour imposer un point de vue.

Le mode de la connotation

21 Cette modalité utilise des paysages-valeur : par un procédé métaphorique, la qualité du paysage est transmise, parfois avec audace, à un produit commercial. C'est généralement le texte qui fait fonctionner la connotation, la signification. Par exemple, il sélectionne la relation d'équivalence parmi toutes celles pouvant exister entre deux images juxtaposées, celle du produit ou du consommateur visé et celle d'un paysage. Sont établies des correspondances valorisantes pour le produit ou celui qui le consomme : un paysage aride et accidenté fournira à une voiture de sport un terrain d'aventure digne de ses qualités mécaniques, ou bien un environnement historique et social raffiné constituera le cadre choisi pour déguster une boisson prestigieuse, etc.

Le mode symbolique

La communication se place délibérément à plusieurs niveaux de signification, conscients et inconscients, comme c'était le cas avec notre village grec. Les paysages apparaissent comme un espace ouvert aux désirs et aux projets. Ils sont le plus souvent exposés et utilisés comme une métonymie du vaste monde et traités selon le registre mythique. Telle montagne est trop montagneuse pour n'être que montagnarde! Elle signifie à la fois l'étrangeté du monde, son hostilité et la grandeur des hommes qui s'y attaquent, etc. Le paysage ne prend forme et sens

qu'en tant qu'acteur d'un drame, d'une épopée, dont il est le théâtre ou l'enjeu ${ }^{4}$. Ce mode fait penser à certaines formes d'utilisation du paysage par la peinture de la Renaissance - Ucello ou Carpaccio, par exemple ${ }^{5}$ - ou de l'époque romantique.

Le mode rhétorique

24 A lui seul, le paysage fonctionne généralement sur le mode connotatif voire symbolique, mais le plus intéressant est, sans doute, la relation qui est établie entre le 
texte et l'image : il y a fréquemment isomorphisme entre l'un et l'autre ; cependant, l'image, tout en disant la même chose que le texte, affirme aussi - assez souvent et dans le même temps - autre chose - voire même le contraire. Par exemple, sur le troisième document, ce pittoresque chemin irlandais confirme la formule volontairement ambiguë «écartez-vous du droit chemin", mais en même temps, il affirme avec force le contraire et c'est comme si l'on ajoutait au texte: "l'aventure oui, mais sans risque, $n i$ même celui de s'ennuyer». (cf. Doc. en annexe. Ecartez-vous du droit chemin.) Cette forme de publicité s'appuie sur la double polysémie du texte et de l'image et sur la possibilité de lectures à plusieurs niveaux. Mais la polysémie plus «capricieuse » moins contrôlable - de l'image produit un effet d'écho ou, plus justement, de miroir, qui tend à inverser le sens sans modifier les lignes. Ajoutons que les récepteurs de ces messages sont généralement plus aptes et plus attentifs à évaluer un texte qu'une image (habitude culturelle que connaissent bien les publicistes et autres manipulateurs d'images). C'est un véritable jeu rhétorique qui s'établit et le plaisir esthétique que procure, éventuellement, ce jeu, provient de la transgression des règles syntaxiques habituelles, conventionnelles, de la communication ${ }^{6}$. Les figures les plus couramment utilisées sont des figures d'élocution et de construction ${ }^{7}$ se situant dans le cadre de la communication établie par le message publicitaire entre un émetteur et un récepteur ciblé. Les tropes ou "figures de mots », et les figures de style peuvent aussi se révéler assez actives dans les discours portés sur le spectacle du monde. Ce traitement du paysage permet d'introduire des niveaux de signification multiples constituant les « harmoniques » du signifié et susceptibles de lui donner une efficacité plus grande, de toucher un public plus large avec un « message » à plus large spectre.

Que retenir de cette typologie schématique? La pratique paysagère de la publicité montre que c'est bien le texte, le discours, qui donne les clés du sens. De quelle manière? Ce n'est pas par une opération de "raffinage ", de "mise en valeur », d'une signification brute qui serait contenue dans le paysage ou dans la sensation que nous en avons, ni par la projection "magique", anthropologique, d'une pensée sur la surface qu'il offre. C'est, semble-t-il, par une opération de sélection du sens que le langage opère dans la polysémie que l'homme projette sur le monde sensible. Cette sélection s'appuie sur les structures qu'il perçoit dans le paysage, un peu comme la pensée s'accroche sur la matérialité (sonore ou lumineuse) des mots ou le calcul mental sur un boulier. Le paysage est constitué en cadre matériel de référence coexistant. En quelque sorte, il donne forme à la pensée. De là vient peut-être la fréquence observée des isomorphismes entre les structures du texte et celles de l'image.

Autrement dit, de façon schématique, une publicité paysagère ou un paysage commenté sont le fruit de cheminements intellectuels qui établissent des relations entre le monde perçu (le réel) et celui des idées, dans un but social ${ }^{8}$ défini. Les sensations "brutes " issues de la perception sont d'abord réduites et constituées en images qualifiées de "paysages». Dans ces images, l'œil et le savoir sélectionnent des formes, des structures, - jugées signifiantes - qui seront mises en relations (selon les divers modes évoqués plus haut) avec des formes ou structures linguistiques, elles-mêmes définies par un discours finalisé issu d'un savoir constitué.

\begin{tabular}{|l|l||l|l|l|l||l|}
\hline Concret & $\begin{array}{l}\text { (concret/ } \\
\text { abstrait) }\end{array}$ & & $\begin{array}{l}\text { (abstrait/ } \\
\text { concret) }\end{array}$ & & abstrait \\
\hline \hline & & & & & & \\
\hline
\end{tabular}




\begin{tabular}{|c|c|c|c|c|c|c|}
\hline Sensation & Image & Formes & & Structures & Discours & Savoir \\
\hline \multirow[t]{4}{*}{ Brute > } & Constituée > & Visibles> & & $<$ Linguistiques & $<$ Finalisé & $<$ Construit \\
\hline & En paysage & & & & & \\
\hline & & & $\begin{array}{l}\text { Imagination } \\
\mathrm{v} \\
\text { Utilisation } \\
\text { sociale }\end{array}$ & & & \\
\hline & & & & $\begin{array}{l}\text { > Opérations } \\
\text { de sélection } \\
\text { réduction } \\
\text { du sens }\end{array}$ & & \\
\hline
\end{tabular}

Ajoutons que, dans le passage de la perception à l'«image active », trois procédures semblent fréquemment mises en œuvre par la publicité paysagère :

- la réduction de la polysémie par le procédé de la redondance qui privilégie un seul niveau de lecture. Le discours tend vers le monosémique.

- a réduction par projection d'une grille formelle, souvent binaire ; c'est un procédé d'information. Le discours tend au fractionnement du sens.

30 - la réduction par la mise en correspondance avec une idée, un sentiment, une sensation, qui mettent le paysage "en perspective»; c'est un procédé d'ordre symbolique qui est utilisé. Le discours tend alors vers une dispersion du sens.

La réduction géographique du paysage

31 L'analyse précédente montre que la publicité met en situation un mode aggloméré placé en objet de désir devant les yeux d'un individu dont toutes les fibres sont sollicitées par la stratégie marchande. Le texte sert à mettre « en tension » la relation la plus complète de l'homme au monde dans l'espace d'un projet autocentré. Elle sait aussi jouer savamment de la dialectique individu/collectivité et, ce faisant, nous éclaire sur la condition existentielle de l'homme habitant ».

Un discours contestable

32 Le « discours géographique », tel que nous l'entendons ici, tend à se placer en retrait du paysage, hors du monde et de ses plaisirs, dans une attitude "quasi monastique ». Il cherche à le découper méthodiquement, selon des «règles canoniques" et stéréotypées ${ }^{9}$, des critères visuels et morphologiques dont l'un des objectifs est de réduire la polysémie, la subjectivité de la perception. Mais, comme dans notre exemple $\mathrm{n}^{\circ} 2$, c'est souvent le choix d'un axe de lecture monosémique fondé sur le postulat morpho-fonctionnaliste qui caractérise l'approche des paysages géographiques la plus répandue.

L'article Paysages II de Maurice RONAI ${ }^{10}$ montre que la grande échelle privilégiée par les paysages valorise le sujet et occulte des enjeux collectifs sous la catégorie esthétique (c'est net avec la publicité) et pose clairement le problème de la «paysagité ». En reliant à la même logique marchande un "espace spéculaire "-qu'il caractérise 
(p. 90) par la visibilité, le plaisir esthétique et le prestige social - à un " espace réel »site, activités, revenus virtuels -, il ouvre une voie de recherche peu fréquentée par la géographie : celui de la force idéologique du paysage. D'où il ressort qu'un discours géographique qui se calque trop sur la logique économique ou, à l'opposé, sur la logique esthétique, participe, d'une manière ou d'une autre, à une occultation d'une part de la réalité. On sait que c'est surtout la logique économique qui a contaminé le discours géographique des trente dernières années. Il conviendrait de lui rendre l'équilibre qu'il faut pour le faire «marcher sur les deux jambes ».

serait donc intéressant d'analyser le langage des géographes lorsqu'il se porte sur l'espace vécu des paysages. On sait que beaucoup de termes descriptifs sont connotés d'anthropomorphisme (croupes, mamelons, cimes hardies...); on sait aussi que le fonctionnalisme explique à bon compte certaines localisations... Cela a été dénoncé. Par contre, on n'a pas analysé comment les géographes introduisent des images, des paysages, dans leurs travaux, comment ils les utilisent, les commentent, les légendent et quels buts ils poursuivent, ce faisant. On n'a pas non plus étudié suivant quelles procédures intellectuelles les géographes découpent les paysages (ou même les cartes) en zones homogènes; c'est aussi un problème d'ordre visuel. Surtout, me semble-t-il, on n'a pas cherché à voir si les structures linguistiques, et les figures de rhétorique qui les constituent, interviennent dans la mise en forme d'un discours sur l'espace. Et, de manière générale, c'est la pertinence scientifique du discours géographique qui se trouve posée si l'on conteste la légitimité des traitements réducteurs qu'il applique aux données de la perception.

Des recherches à poursuivre

Soufflée par l'analyse de la pratique publicitaire des paysages, notre hypothèse de travail consiste à supposer que le discours géographique, loin d'être toujours rigoureux lorsqu'il s'applique aux paysages, se laisse contaminer par ce qui constitue, consciemment ou non, son projet social et que celui-ci peut être mis en évidence, notamment par l'étude des réductions ou occultations de sens qu'opère la stratégie linguistique mise en œuvre. Ce qui revient à traquer les signes de la dérive idéologique d'un discours.

Cela conduirait, par exemple, à étudier :

37 - les isomorphismes entre paysages étudiés et commentaires portés sur eux ;

38 - le recours à des figures telles que la métonymie, la synecdoque, le pléonasme, la redondance, le zeugma... la liste serait longue ${ }^{11}$, et leurs effets ;

39 - le « lexique sémiotique » qu'utilisent les géographes, c'est-à-dire ce qui, pour eux, fait signe dans un paysage, ce qu'ils y cherchent et ce qu'ils négligent ;

40 - les niveaux de lecture choisis et surtout les glissements qui s'y produisent notamment dans la dialectique monosémie-polysémie ;

41 - les types de paysages utilisés et le rôle qui leur est dévolu par rapport au cheminement textuel : je pense notamment à la pratique qui consiste à ériger certains paysages en idéal-type, en archétypes ou en formes symboliques, ce qui ne revient jamais au même.

42 Ce serait poursuivre le travail d'auto-analyse que la géographie a commencé à faire, il y a une vingtaine d'années, et approfondir dans cette voie la réflexion épistémologique dont elle a besoin. Mais ce serait aussi lui ouvrir d'autres voies qui sont à sa portée.

Vers un territoire des images. 

contraire, à son retour "en force» dans ce domaine de recherche. Plusieurs géographes ont déjà ouvert des pistes nouvelles dans cette direction mais il n'est pas question de les évoquer ici. Bornons-nous à signaler que ces travaux se sont orientés dans trois grandes directions : l'une, assez technicienne, consisterait à rechercher les bases d'une analyse scientifique du paysage (indicateurs paysagers) dont ont besoin les aménageurs et les pouvoirs publics ; une autre, plus critique, avec L. UZAN ${ }^{12}$, m. RONAI et "l'équipe " d'Hérodote dénonce vivement les ambiguïtés idéologiques de la " paysagité »; la troisième est plus sensible à l'inflexion " humaniste » de la géographie contemporaine. Dans cette dernière approche, le travail d'A. BERQUE est particulièrement intéressant. Dans Le Sauvage et l'Artifice ${ }^{13}$ et ailleurs ${ }^{14}$, il tend à nier la pure objectivité comme la pure subjectivité du paysage pour aboutir au nouveau concept géographique de " trajet ", permettant d'" explorer ces entre-deux - entre le poursoi de la conscience et l'en-soi de la chose... ». Cela rejoint les travaux de MERLEAU-PONTY, DARDEL, LEDRUT, SANSOT et d'autres: une certaine convergence des différentes sciences humaines nous conduit donc à situer - ou à étendre - l'objet de la géographie à la relation complexe qui, entre l'ensemble matière-étendue (le monde en soi) et l'ensemble individu-société (l'humanité), place le monde du langage et celui des formes signifiantes dans le sens où LEDRUT emploie ce terme ${ }^{15}$ (c'est-à-dire des échangeurs de substance abstraite et concrète, dirons-nous, pour aller vite). l'amplification des images mentales à l'œuvre dans l'opinion publique et la culture de masse ; la publicité y a sa part, plus que la géographie. Ces images mentales dont les paysages font de plus en plus partie peuvent constituer des facteurs non négligeables d'action sur le milieu géographique. Par exemple, l'aménagement d'un territoire, quel qu'il soit, suppose sa délimitation, son arpentage, son maillage et ses équipements, c'est entendu; mais il suppose aussi une légitimation de l'ordre que l'on y installe ou de l'idéologie que l'on y véhicule; ce que RONAI appelle la "mise en scène de l'espace». Il en va de même pour tous les procès de (dé-, re-) territorialisation. Le contrôle territorial s'appuie autant, sinon plus aujourd'hui, sur un arsenal idéologique que sur des équipements ou des actions de surveillance et d'administration; le territoire serait, de plus en plus, un sens donné à l'espace. Il serait le fruit de "mécanismes " mentaux collectivisés par ceux qui, détenant le pouvoir de créer, de modifier et de diffuser des images mentales, sont aussi en mesure d'en tirer profit, gloire ou pouvoir. C'est tout cela que recouvre, en fait, le terme d'« imaginaction » introduit plus haut. La politique d'image de marque de villes telles que Grenoble ou Toulouse ou de régions telles que le Nord en serait l'illustration ${ }^{16}$; ces actions médiatiques jouent un grand rôle dans un contexte économique de re-localisation industrielle et financière. A la manière de la publicité, ces actions, ces images occultant les conflits d'intérêts ou de valeurs, forcent l'adhésion, et produisent du consensus.

La géographie, en son temps, a fourni des cadres mentaux qui sont encore actifs aujourd'hui - "vocations touristiques ou agricoles", "sites privilégiés", "centre historique » - et joué, ce faisant, un rôle idéologique et technocratique non négligeable. Aujourd'hui, certaines représentations de l'espace - je pense aux chorêmes ou à certains modèles spatiaux - en offrent des images dont on doit se demander, lorsqu'on les utilise, si elles ne sont pas trop réductrices. Mais ajoutons aussitôt que la géographie dispose aussi des meilleurs moyens qui soient pour contester, si besoin est, ces « images

Strates, 4 | 1989 
qui font des espaces", ces paysages qui font des vocations... etc. Les récents travaux d'épistémologie de la discipline ${ }^{17}$ préparent le terrain d'une évolution de son utilité sociale vers l'analyse des pratiques idéologiques appliquées à la dimension spatiale. Et, dans ce domaine, le recours au concept d'imaginaction suppose que la pensée géographique s'ouvre à une approche moins réductrice de l'espace vécu et, pour ce faire, qu'elle s'intéresse notamment aux procédures logiques, linguistiques ou esthétiques qui contribuent à façonner la réalité géographique.

\section{NOTES}

1. Il importe de préciser que ce que nous appellerons ici « le discours des géographes » n'est pas la somme ou le détail du savoir géographique spécialisé ; il s'agit plutôt de ce discours second, dérivé, de ce « savoir »- plus flou mais aussi plus répandu dans l'opinion - que crée le processus de vulgarisation ou de médiatisation, notamment par le biais de l'enseignement, des manuels scolaires, de certaines revues, etc.

2. COLLOT (M.). Points de vue sur la perception des paysages. L'Espace Géographique, $1986, \mathrm{n}^{\circ} 3$.

3. Ceci a fait l'objet d'un travail plus détaillé dans un mémoire de D.E.A. préparé à l'Université de Paris-I sous la direction de P. PINCHEMEL au cours de l'année 1988 (DEA Analyse théorique et épistémologie en géographie).

4. Cela a été analysé par FOUCHER (M.), « Du désert, paysage de western », Hérodote, $\mathrm{n}^{\circ} 7,1977$ et $\mathrm{n}^{\circ} 44,1987$.

5. 5 Cf. l'article de J. PETITOT-COCORDA, « Saint-Georges, Remarques sur l'espace pictural », in Sémiotique de l'Espace, Médiations Denoël-Gonthier, 1979.

6. C'est ce que pensent VICTOROFF (D.), La publicité et l'image, Médiations, DenoëlGonthier, Paris, 1978, (dont je me suis librement inspiré ici), DURAND (J.), « Rhétorique et image publicitaire », Communications, $n^{\circ} 15,1970$, ainsi que BARTHES (R.), «Eléments de sémiologie ", Communications, $\mathrm{n}^{\circ} 4,1964$.

10 RONAI (M.), Paysages II, Hérodote, $n^{\circ} 7,1977$.

7. La terminologie utilisée est celle de FONT AMER (P.), Les figures du discours, "Champs ", n 15, Flammarion, 1977.

8. Le mot est à prendre ici au sens large.

9. Souvent issues de la géomorphologie classique ou «structuraliste ».

10. RONAI (M.), « Paysages II », Hérodote, $n^{\circ} 7,1977$.

11. FONTANIER (P.), op. cit.

12. UZAN (L.), Cadre de vie et décors de récréation, I.R.U, Université Paris-XII, Juin 1981.

13. BERQUE (A.), Le Sauvage et l'artifice, Paris, Gallimard, 1986.

14. BERQUE (A.), « Milieu, médiane et médialité », L'espace géographique, 1987, $\mathrm{n}^{\circ} 4$.

15. LEDRUT (R.), La Forme et le Sens dans la Société, Méridiens, 1985.

16. PUMAIN (D.) City image in an urban system, $29^{\mathrm{e}}$ Congrès Européen de l'Association de Science Régionale, Cambridge, 29 août- $1^{\mathrm{er}}$ septembre 1989 et COHEN (J.), GUERRINI (MC.), MATHIEU (N.) et ROBIC (M-C) «L'iconographie dans l'aménagement du territoire : 
changer la donne et/ou donner le change ? ", Colloque de géographie politique : L'Etat et les stratégies du territoire hier et aujourd'hui, Paris, 29-30 mai 1989.

17. Ceux du groupe « Histoire et épistémologie de la géographie » URA 914, CNRS/

Paris-I, en particulier.

\section{RÉSUMÉS}

Le commentaire d'un paysage est la mise en forme verbale de perceptions: c'est une information du monde. L'analyse des pratiques « paysagères » de la publicité montre que la création du sens résulte de l'action opérée sur les images par les mécanismes mimétiques, symboliques et rhétoriques d'un discours finalisé. L'approche géographique des paysages consiste souvent à créer ou orienter des "points de vue » qui réduisent la polysémie des images. Ce mécanisme d' " imaginaction", susceptible d'influencer certaines pratiques territoriales, mérite d'être étudié. Il fonde, en effet, le pouvoir social de la géographie, qu'il soit de nature scientifique ou idéologique. Il convient donc que les géographes s'intéressent à la manière dont « on fait parler les images » et qu'ils commencent par étudier leur propre discours.

Advertising and geography : landscapes, images and discurses

The commentary of a landscape is a transcription of perceptions : it is an "information" of the world. The practices used in advertising are helpful to understand how, to make sense, images hâve to be associated with incentive statements, both operating through mimetic, symbolic and rhetoric références. The geographical approach of landscapes often consists in creating or selecting choosen "points of view" and, as a result, in limiting the polysemy of images. The way "imaginaclion" is able to partly influence some spatial practices is worth being considered. Indeed, it might bring to geography its social power whether on scientific or idcological grounds. Consequently, geographers ought to be aware of various uses and practices of images and get a new glance at their own statements.

\section{INDEX}

Mots-clés : paysage, publicité, rhétorique, discours géographique, « imaginaclion »

Keywords : landscape, advertising, discurse, geographical discurse, \&quot, imaginaction\&quot

\section{AUTEUR}

\section{DIDIER MENDIBIL}

Professeur agrégé de géographie, enseignant à l'Ecole Normale de Melun et président de l'Association GROPIUS qui prépare un atlas de Seine et Marne. Il travaille sur la dimension idéologique des images mentales en géographie et a écrit, notamment, une étude sur Le sentiment d'insécurité dans le centre de Rouen, à paraître dans Etudes Normandes. 